\title{
Existence and uniqueness for a class of iterative fractional differential equations
}

\author{
Rabha W Ibrahim ${ }^{1}$, Adem Kılıçman ${ }^{2 *}$ and Faten H Damag ${ }^{2}$
}

"Correspondence:

akilic@upm.edu.my

${ }^{2}$ Department of Mathematics,

University Putra Malaysia, Serdang,

Malaysia

Full list of author information is

available at the end of the article

\begin{abstract}
The presence of a self-mapping increases the difficulty in proving the existence and uniqueness of solutions for general iterative fractional differential equations. In this article, we provide conditions for the existence and uniqueness of solutions for the initial value problem. We also determine the Burton stability of such equations. The arbitrary order case is taken in the sense of Riemann-Liouville fractional operators.
\end{abstract}

\section{Introduction}

Fractional calculus is an area of specialization in mathematics that is concerned with differential and integral operators of arbitrary orders. Fractional derivatives are considered excellent tools for the description of nonlinearity. Fractional derivatives are mainly used with classical integer order patterns, in which such effects are neglected. The significance of fractional derivatives is manifested in modeling, electrical properties of real materials, capacity of rheological properties of rocks, and many other fields. Fractional derivatives and fractional integrals have various definitions. The most famous operators are Riesz, Grunwald-Letnikov, Riemann-Liouville, and Caputo [1-10].

Existence and uniqueness theory is studied widely in fractional differential equations. Agarwal et al. studied the existence of solutions for the Riemann-Liouville operator for a class of integro-differential equations of high fractional order [11]. Moreover, Agarwal et al. presented some existence and uniqueness outcomes for a class of fuzzy fractional integral equations utilizing the Schauder fixed point theorem in semilinear Banach spaces [12]. Li et al. established the existence of a class of nonlinear fractional $q$-difference equations with mixed nonlinear assumptions, including the fractional $q$-derivative of Riemann-Liouville type, employing the Guo-Krasnoselskii fixed point theorem on cones [13]. Ibrahim applied the Tarski fixed point theorem to study the existence of extremal solutions for fractional differential equations with maxima [14]. The existence and uniqueness in complex domains have been imposed considering the Srivastava-Owa fractional operators $[15,16]$.

In this study, we introduce some conditions for the existence and uniqueness of solutions for the initial value problems of iterative equations. We also determine the Burton stability of such equations. The arbitrary order case is taken in the sense of Riemann-Liouville fractional operators, as well as Caputo derivative.

\section{Springer}

(c) 2015 Ibrahim et al.; licensee Springer. This is an Open Access article distributed under the terms of the Creative Commons Attribution License (http://creativecommons.org/licenses/by/4.0), which permits unrestricted use, distribution, and reproduction in any medium, provided the original work is properly credited. 


\section{Preliminaries}

This section deals with some preliminaries and notations regarding fractional calculus.

Fractional calculus originated from the Riemann-Liouville definition of fractional integral of order $\alpha$ in the form

$$
I_{a}^{\alpha} \psi(t)=\int_{a}^{t} \frac{(t-\tau)^{\alpha-1}}{\Gamma(\alpha)} f(\tau) d \tau
$$

where $\psi$ is continuous.

The fractional (arbitrary) order differential of the continuous function $\psi$ of order $\alpha>0$ is defined by

$$
D_{a}^{\alpha} \psi(t)=\frac{d}{d t} \int_{a}^{t} \frac{(t-\tau)^{-\alpha}}{\Gamma(1-\alpha)} \psi(\tau) d \tau
$$

When $a=0$, we conclude that

$$
D^{\alpha} t^{m}=\frac{\Gamma(m+1)}{\Gamma(m-\alpha+1)} t^{m-\alpha}, \quad m>-1 ; 0<\alpha<1
$$

and

$$
I^{\alpha} t^{m}=\frac{\Gamma(m+1)}{\Gamma(m+\alpha+1)} t^{m+\alpha}, \quad m>-1 ; \alpha>0 .
$$

Let $\psi$ be a continuously ( $n$ ) differentiable function. The Caputo derivative can then be realized by the following form:

$$
D_{t}^{\gamma} f(t)=\frac{1}{\Gamma(n-\gamma)} \int_{a}^{t} \frac{\psi^{(n)}(\zeta)}{(t-\zeta)^{\gamma-n+1}} d \zeta, \quad(n-1) \leq \gamma<n,
$$

where $n$ is an integer and $\gamma$ is a real number.

\section{Related work}

Differential equations with state-dependent delays attract interest of specialists because they arise from application models, such as the two-body problem of classical electrodynamics, transmission, mechanical models, population models, infection disease, position control, and dynamics of economical systems. As a special type of state-dependent delay differential equations, iterative differential equations have distinct characteristics. Such equations have been investigated in recent years in terms of their smoothness, analyticity, monotonicity, convexity, and numerical solution. In the theory of differential equations, one of the essential and important problems is the initial value problem. Numerous existence results on special iterative differential equations can be found in the literature. In 1984, Eder proved the existence of the unique monotone solution for the second iterative differential equation [17] using the contraction principle as follows:

$$
y^{\prime}(t)=y(y(t))
$$


linked with $y\left(t_{0}\right)=t_{0}\left(t_{0} \in[-1,1]\right)$. Fečkan investigated the general second iterative differential equation [18]

$$
y^{\prime}(t)=f(y(y(t)))
$$

with the initial value $y(0)=0$. Modified iterative functional differential equations have been recently suggested by Zhang and Gong [19] to determine approximate solutions. They studied the following iterative functional differential equation:

$$
\begin{aligned}
& y^{\prime}(t)=f\left(t, y^{[1]}(t), y^{[2]}(t), \ldots, y^{[n]}(t)\right), \\
& y\left(t_{0}\right)=y_{0},
\end{aligned}
$$

which satisfies the following result.

Lemma 3.1 Let

$$
\varphi_{M}=\left\{y \in C^{0}\left(\left[t_{0}-h, t_{0}+h\right]\right):|y(t)-y(s)| \leq M|t-s|, \forall t, s \in\left[t_{0}-h, t_{0}+h\right]\right\},
$$

where $M<1$. Iff, $g \in \varphi_{M}$, then

$$
\left\|f^{[i]}-g^{[i]}\right\|_{\left[t_{0}-h, t_{0}+h\right]} \leq \frac{1-M^{i}}{1-M}\|f-g\|_{\left[t_{0}-h, t_{0}+h\right]}, \quad i=1,2, \ldots .
$$

Ibrahim utilized nonexpansive operators to establish the existence and uniqueness of iterative fractional differential equations of various forms [20, 21]. Recently, Ibrahim and Darus established sufficient conditions for fractional differential equations as follows:

$$
D^{\gamma} y(t)=f(t, y(t), y(\beta t), y(y(t))), \quad \beta \in(0,1]
$$

with the initial value $y(0)=y_{0}$, see [22].

In this study, we aim to establish the existence and uniqueness of the fractional iterative

$$
D^{\gamma} y(t)=f\left(t, y^{[1]}(t), y^{[2]}(t), \ldots, y^{[n]}(t)\right)
$$

subject to the initial value

$$
y\left(t_{0}\right)=y_{0}
$$

where $y^{[j]}(t):=y\left(y^{[j-1]}(t)\right)$ indicates the $j$ th iterate of self-mapping $y$, where $j=1,2, \ldots, n$.

\section{Existence and uniqueness}

We start with the following result.

Theorem 4.1 Suppose that $f: \Re^{n+1} \rightarrow \Re$ is continuous. If positive r exists, such that

$$
\left(1-M_{1}\right) r \geq l_{0}, \quad l_{0}>0,
$$

where $M_{1}=\|f\|_{\bar{B}\left(z_{0}, r\right)} \leq 1$ and $\bar{B}\left(z_{0}, r\right)$ denotes the closed ball centered at $z_{0}=\left(t_{0}, y_{0}, \ldots, y_{0}\right)$ with radius $r$, then Eq. (1) has a solution defined on $\left[t_{0}-l, t_{0}+l\right]$ for any $l \in\left[l_{0} /\left(1-M_{1}\right), r\right]$. 
Proof The existence of solutions of Eq. (1) is equivalent to finding a continuous solution of the integral equation

$$
y(t)=y_{0}+\int_{0}^{t} f\left(s, y^{[1]}(s), y^{[2]}(s), \ldots, y^{[n]}(s)\right) \frac{(t-s)^{\gamma-1}}{\Gamma(\gamma)} d s .
$$

Define a set $\varphi_{M_{1}}$ as follows:

$$
\begin{aligned}
\varphi_{M_{1}}= & \left\{y \in C^{0}\left(\left[t_{0}-l, t_{0}+l\right]\right): y\left(t_{0}\right)=y_{0},|y(t)-y(s)| \leq M_{1} \frac{|t-s|^{\gamma}}{\Gamma(\gamma+1)},\right. \\
& \left.\forall t, s \in\left[t_{0}-l, t_{0}+l\right]\right\}
\end{aligned}
$$

for any $l \in\left[l_{0} /\left(1-M_{1}\right), r\right]$. Then, for $y \in \varphi_{M_{1}}$, we prove that $y^{[j]}(t)(j=2,3, \ldots, n)$ are well defined on $\left[t_{0}-l, t_{0}+l\right]$. It suffices to establish

$$
\left|y^{[j]}(t)-y_{0}\right| \leq l
$$

for $j \in \mathbb{N}$ by induction. In fact, for $t_{0} \leq s \leq t$, we have

$$
\left|y(t)-y_{0}\right| \leq|y(t)-y(s)|+\left|y(s)-y_{0}\right| \leq M_{1} l+M_{1} \frac{|t-s|^{\gamma}}{\Gamma(\gamma+1)} \leq l .
$$

We let $\left|y^{[j]}(t)-y_{0}\right| \leq l$ for a positive integer $j \geq 1$, then

$$
\left|y^{[j+1]}(t)-y_{0}\right| \leq M_{1}\left|y^{[j]}(t)-y(s)\right|+\left|y(s)-y_{0}\right| \leq l \text {. }
$$

On the basis of induction, (4) holds and $y^{[j]}\left(\left[t_{0}-l, t_{0}+l\right]\right)$ are well defined for any $y \in \varphi_{M_{1}}$. In the sequel we apply the Schauder fixed point theorem to prove the existence of the continuous solution of Eq. (3). To this end, we define the integral operator $\mathcal{G}: \varphi_{M_{1}} \rightarrow$ $C^{0}\left(\left[t_{0}-l, t_{0}+l\right]\right)$ by

$$
\mathcal{G} y(t):=y_{0}+\frac{1}{\Gamma(\gamma)} \int_{0}^{t} f\left(s, y^{[1]}(s), y^{[2]}(s), \ldots, y^{[n]}(s)\right)(t-s)^{\gamma-1} d s
$$

Clearly,

$$
\mathcal{G} y\left(t_{0}\right)=y_{0}+\frac{1}{\Gamma(\gamma)} \int_{0}^{t_{0}} f\left(s, y^{[1]}(s), y^{[2]}(s), \ldots, y^{[n]}(s)\right)(t-s)^{\gamma-1} d s
$$

for any $y \in \varphi_{M_{1}}$. In view of

$$
\begin{aligned}
& \left\|\left(t, y^{[1]}(t), y^{[2]}(t), \ldots, y^{[n]}(t)\right)-\left(t_{0}, y_{0}, y_{0}, \ldots, y_{0}\right)\right\| \\
& \quad=\max \left\{\left|t-t_{0}\right|,\left|y^{[1]}(t)-y_{0}\right|,\left|y^{[2]}(t)-y_{0}\right|, \ldots,\left|y^{[n]}(t)-y_{0}\right|\right\} \\
& \quad \leq \max \left\{\left|t-t_{0}\right|, M_{1}\left|t-t_{0}\right|, M_{1}\left|y^{[1]}(t)-y_{0}\right|, \ldots, M_{1}\left|y^{[n-1]}(t)-y_{0}\right|\right\} \\
& \quad \leq \max \left\{l, M_{1} l, M_{1} l, \ldots, M_{1} l\right\} \leq l \leq r,
\end{aligned}
$$


we get

$$
\begin{aligned}
\left|\mathcal{G} y\left(t_{1}\right)-\mathcal{G} y\left(t_{2}\right)\right| \leq & \left|\int_{0}^{t_{1}} f\left(s, y^{[1]}(s), y^{[2]}(s), \ldots, y^{[n]}(s)\right)\right| \frac{\left(t_{1}-s\right)^{\gamma-1}}{\Gamma(\gamma)} d s \\
& -\int_{0}^{t_{2}} f\left(s, y^{[1]}(s), y^{[2]}(s), \ldots, y^{[n]}(s)\right)\left|\frac{\left(t_{2}-s\right)^{\gamma-1}}{\Gamma(\gamma)} d s\right| \\
\leq & M_{1} \frac{\left|t_{1}-t_{2}\right|^{\gamma}}{\Gamma(\gamma+1)}, \quad t_{1}, t_{2}>s .
\end{aligned}
$$

Then $\varphi_{M_{2}}$ is a nonempty convex and compact subset of the Banach space $C^{0}([a, b])$. We consider the mapping $\mathcal{T}: \varphi_{M_{2}} \rightarrow C^{0}([a, b])$ defined by

$$
\mathcal{T} y(t):=y_{0}+\frac{1}{\Gamma(\gamma)} \int_{0}^{t} f\left(s, y^{[1]}(s), y^{[2]}(s), \ldots, y^{[n]}(s)\right)(t-s)^{\gamma-1} d s .
$$

To show $\mathcal{T}$ is a self-mapping, we note that for any $t_{1}, t_{2} \in\left[t_{0}-l, t_{0}+l\right]$. Therefore Eqs. (5)-(7) yield $\mathcal{G} y \in \varphi_{M_{1}}$; (i.e., $\mathcal{G}$ is a self-mapping operator). It remains to show that $\mathcal{G}$ is continuous. For this purpose, taking any $y_{1}, y_{2} \in \varphi_{M_{1}}$, we obtain

$$
\begin{aligned}
\left|\mathcal{G} y_{1}(t)-\mathcal{G} y_{2}(t)\right| \leq & \int_{0}^{t} \mid f\left(s, y_{1}^{[1]}(s), y_{1}^{[2]}(s), \ldots, y_{1}^{[n]}(s)\right) \\
& -f\left(s, y_{2}^{[1]}(s), y_{2}^{[2]}(s), \ldots, y_{2}^{[n]}(s)\right) \mid \frac{(t-s)^{\gamma-1}}{\Gamma(\gamma)} d s, \quad t>s .
\end{aligned}
$$

By Lemma 3.1, we conclude that

$$
\begin{aligned}
\| & \left(s, y_{1}^{[1]}(s), y_{1}^{[2]}(s), \ldots, y_{1}^{[n]}(s)\right)-\left(s, y_{2}^{[1]}(s), y_{2}^{[2]}(s), \ldots, y_{2}^{[n]}(s)\right) \| \\
& =\max \left\{\left|y_{1}^{[1]}(s)-y_{2}^{[1]}(s)\right|,\left|y_{1}^{[2]}(s)-y_{2}^{[2]}(s)\right|, \ldots,\left|y_{1}^{[n]}(s)-y_{2}^{[n]}(s)\right|\right\} \\
& \leq \max \left\{\left\|y_{1}-y_{2}\right\|_{\left[t_{0}-l, t_{0}+l\right]}, \frac{1-M_{1}^{2}}{1-M_{1}}\left\|y_{1}-y_{2}\right\|_{\left[t_{0}-l, t_{0}+l\right]}, \ldots, \frac{1-M_{1}^{n}}{1-M_{1}}\left\|y_{1}-y_{2}\right\|_{\left[t_{0}-l, t_{0}+l\right]}\right\} \\
& =\frac{1-M_{1}^{n}}{1-M_{1}}\left\|y_{1}-y_{2}\right\|_{\left[t_{0}-l, t_{0}+l\right]}<\frac{1}{1-M_{1}}\left\|y_{1}-y_{2}\right\|_{\left[t_{0}-l, t_{0}+l\right] .}
\end{aligned}
$$

Given the uniform continuity of $f$ on $\bar{B}\left(z_{0}, r\right)$, for any $\varepsilon>0$, there exists $\delta(\varepsilon)>0$, the inequality

$$
\left\|\mathcal{G} y_{1}-\mathcal{G} y_{2}\right\|<\varepsilon l
$$

holds for $\left\|y_{1}-y_{2}\right\|_{\left[t_{0}-l, t_{0}+l\right]}<\delta$, which yields $\mathcal{G}$ is continuous. $\Phi_{M_{1}}$ is a convex, compact subset of the Banach space $C^{0}\left(\left[t_{0}-l, t_{0}+l\right]\right)$ and $\mathcal{G}$ is a continuous operator that satisfies all conditions of the Schauder fixed point theorem, $\mathcal{G}$ has a fixed point $g \in \varphi_{M_{1}}$ and $g$ is a solution for Eq. (1) on the interval $\left[t_{0}-l, t_{0}+l\right]$. This completes the proof.

Theorem 4.2 Suppose that $f: \Re^{n+1} \rightarrow \Re$ is continuous and any compact interval $[a, b]$ includes $t_{0}$ and $y_{0}$. If

$$
M_{2} A_{t_{0}} \leq B_{y_{0}}
$$


where $A_{t_{0}}=\max \left\{t_{0}-a, b-t_{0}\right\}, B_{y_{0}}=\min \left\{y_{0}-a, b-y_{0}\right\}, M_{2}=\|f\|_{[a, b]^{n+1}}$ and $[a, b]^{n+1}$ denotes the product of $n+1$ intervals $[a, b]$, then Eq. (1) has a solution defined on $[a, b]$.

Proof Let

$$
\varphi_{M_{2}}=\left\{y \in C^{0}([a, b],[a, b]): y\left(t_{0}\right)=y_{0},|y(t)-y(s)| \leq M_{2} \frac{|t-s|^{\gamma}}{\Gamma(\gamma+1)}, \forall t, s \in[a, b]\right\},
$$

then $\varphi_{M_{2}}$ is a nonempty convex and compact subset of the Banach space $C^{0}([a, b])$. We consider the mapping $\mathcal{T}: \varphi_{M_{2}} \rightarrow C^{0}([a, b])$ to be defined by

$$
\mathcal{T} y(t):=y_{0}+\frac{1}{\Gamma(\gamma)} \int_{0}^{t} f\left(s, y^{[1]}(s), y^{[2]}(s), \ldots, y^{[n]}(s)\right)(t-s)^{\gamma-1} d s
$$

To show $\mathcal{T}$ is a self-mapping, we note that

$$
\begin{aligned}
|\mathcal{T} y(t)| & \leq y_{0}+\frac{1}{\Gamma(\gamma)}\left|\int_{0}^{t} f\left(s, y^{[1]}(s), y^{[2]}(s), \ldots, y^{[n]}(s)\right)(t-s)^{\gamma-1} d s\right|, \quad t>s \\
& \leq y_{0}+M_{2} \frac{\left(t-t_{0}\right)^{\gamma}}{\Gamma(\gamma+1)}, \quad t>t_{0} \\
& \leq y_{0}+M_{2} A_{t_{0}} \leq y_{0}+B_{t_{0}} \leq b, \\
|\mathcal{T} y(t)| & \geq y_{0}-\frac{1}{\Gamma(\gamma)}\left|\int_{0}^{t} f\left(s, y^{[1]}(s), y^{[2]}(s), \ldots, y^{[n]}(s)\right)(t-s)^{\gamma-1} d s\right|, \quad t>s \\
& \geq y_{0}-M_{2} \frac{\left|t-t_{0}\right|^{\gamma}}{\Gamma(\gamma+1)} \geq y_{0}-M_{2} A_{t_{0}} \\
& \geq y_{0}-B_{y_{0}} \geq a .
\end{aligned}
$$

Clearly, $\mathcal{T} y\left(t_{0}\right)=y_{0}$. Moreover, for any $t_{1}, t_{2} \in[a, b]$, we obtain

$$
\begin{aligned}
\left|\mathcal{T} y\left(t_{1}\right)-\mathcal{T} y\left(t_{2}\right)\right| & \leq \frac{1}{\Gamma(\gamma)} \int_{t_{2}}^{t_{1}}\left|f\left(s, y_{1}^{[1]}(s), y_{1}^{[2]}(s), \ldots, y_{1}^{[n]}(s)\right)\right|(t-s)^{\gamma-1} d s, \quad t>s \\
& \leq M_{2} \frac{\left|t_{1}-t_{2}\right|^{\gamma}}{\Gamma(\gamma+1)} .
\end{aligned}
$$

Therefore, (12) and (13) yield that $\mathcal{T}$ maps $\varphi_{M_{2}}$ into itself. The definitions of $A_{t_{0}}$ and $B_{y_{0}}$ prove that $M_{2} \leq 1$, thus for any $y_{1}, y_{2} \in \varphi_{M_{2}}$, according to Lemma 3.1, we have

$$
\begin{aligned}
\| & \left(s, y_{1}^{[1]}(s), y_{1}^{[2]}(s), \ldots, y_{1}^{[n]}(s)\right)-\left(s, y_{2}^{[1]}(s), y_{2}^{[2]}(s), \ldots, y_{2}^{[n]}(s)\right) \| \\
& =\max \left\{\left|y_{1}^{[1]}(s)-y_{2}^{[1]}(s)\right|,\left|y_{1}^{[2]}(s)-y_{2}^{[2]}(s)\right|, \ldots,\left|y_{1}^{[n]}(s)-y_{2}^{[n]}(s)\right|\right\} \\
& \leq \max \left\{\left\|y_{1}-y_{2}\right\|_{[a, b]}, \frac{1-M_{2}^{2}}{1-M_{2}}\left\|y_{1}-y_{2}\right\|_{[a, b]}, \ldots, \frac{1-M_{2}^{n}}{1-M_{2}}\left\|y_{1}-y_{2}\right\|_{[a, b]}\right\} \\
& =\frac{1-M_{2}^{n}}{1-M_{2}}\left\|y_{1}-y_{2}\right\|_{[a, b]} \\
& <\frac{1}{1-M_{2}}\left\|y_{1}-y_{2}\right\|_{[a, b]} .
\end{aligned}
$$


By the uniform continuity of $f$ on $[a, b]^{n+1}$, for any $\varepsilon>0$, there exists $\delta(\varepsilon)>0$. When $\| y_{1}-$ $y_{2} \|_{[a, b]}<\delta$, we obtain

$$
\left|f\left(s, y_{1}^{[1]}(s), y_{1}^{[2]}(s), \ldots, y_{1}^{[n]}(s)\right)-f\left(s, y_{2}^{[1]}(s), y_{2}^{[2]}(s), \ldots, y_{2}^{[n]}(s)\right)\right|<\varepsilon
$$

Consequently,

$$
\begin{aligned}
\left|\mathcal{T} y_{1}(t)-\mathcal{T} y_{2}(t)\right| \leq & \int_{0}^{t} \mid f\left(s, y_{1}^{[1]}(s), y_{1}^{[2]}(s), \ldots, y_{1}^{[n]}(s)\right) \\
& -f\left(s, y_{2}^{[1]}(s), y_{2}^{[2]}(s), \ldots, y_{2}^{[n]}(s)\right) \mid \frac{(t-s)^{\gamma-1}}{\Gamma(\gamma)} d s, \quad t>s \\
< & \varepsilon(b-a),
\end{aligned}
$$

which means that $\mathcal{T}$ is a continuous operator. It follows that $\varphi_{M_{2}}$ is a convex, compact subset of the Banach space $C^{0}([a, b])$. By the Schauder fixed point theorem, $\mathcal{T}$ has a fixed point $h \in \varphi_{M_{2}}$ and $h$ is a solution of Eq. (1) on the interval $[a, b]$. This completes the proof.

\section{Applications}

In this section, our theorems are illustrated by the following example. First, we prove the existence of smooth solutions of (1) based on the results provided by Si and Wang [23]. In this paper, smooth function $g \in C^{n}$ means that the function $g$ has a number of continuous derivatives, and its $n$th continuous derivative is Lipschitzian. We require the following result.

Lemma 5.1 Let

$$
\begin{aligned}
\Omega\left(N_{1}, \ldots, N_{n+1} ; I\right)= & \left\{g \in C^{n}(I, I):\left|g^{(i)}(t)\right| \leq N_{i}, i=1,2, \ldots, n\right. \\
& \left.\left|g^{(n)}(t)-g^{(n)}(s)\right| \leq N_{n+1}|t-s|, t, s \in I\right\} .
\end{aligned}
$$

Then, for any $x(t) \in \Omega\left(N_{1}, \ldots, N_{n+1} ; I\right)$, there exist a function

$$
y_{* i k}(t)=P_{i k}\left(y_{10}(t), \ldots, y_{1, i-1}(t) ; \ldots ; y_{k 0}(t), \ldots, y_{k, i-1}(t)\right)
$$

and positive constants $N_{u v}^{i k}$ such that

$$
\left|P_{i k}\left(\bar{\lambda}_{10}, \ldots, \bar{\lambda}_{k, i-1}\right)-P_{i k}\left(\tilde{\lambda}_{10}, \ldots, \tilde{\lambda}_{k, i-1}\right)\right| \leq \sum_{u=1}^{k} \sum_{v=0}^{i-1} N_{u v}^{i k}\left|\bar{\lambda}_{u v}-\tilde{\lambda}_{u v}\right|
$$

for $\left(\bar{\lambda}_{10}, \ldots, \bar{\lambda}_{k, i-1}\right),\left(\tilde{\lambda}_{10}, \ldots, \tilde{\lambda}_{k, i-1}\right)$ belong to compact sets $\left[0, N_{1}\right]^{i} \times\left[0, N_{2}\right]^{i} \times \cdots \times\left[0, N_{k}\right]^{i}$, where $y_{r i}(t)=y^{(r)}\left(y^{[i]}(t)\right), y_{* i k}(t)=\left(y^{[i]}(t)\right)^{(k)}$ and $P_{i k}$ is a uniquely defined multivariate polynomial with nonnegative coefficients and $1 \leq u \leq k, 0 \leq v \leq i-1$.

Theorem 5.1 Consider the equation

$$
D^{\gamma} y(t)=\sum_{i=1}^{m} a_{i}(t) y^{[i]}(t)+F(t)
$$

associated with $y\left(t_{0}\right)=y_{0}$, where $a_{i}(t), F(t) \in C^{n}$ are given smooth functions. 
Proof For $R>0$, by the smoothness of the given functions, we have positive $M_{a_{j}}$ and $M_{F}$ such that

$$
\left|a_{i}(t)\right| \leq M_{a_{i}}, \quad|F(t)| \leq M_{F}, \quad t \in\left[t_{0}-R, t_{0}+R\right], i=1,2, \ldots, m .
$$

Denote

$$
M_{a}=\max _{1 \leq i \leq m}\left\{M_{a_{i}}\right\}, \quad N_{1}=m M_{a}\left(\left|t_{0}\right|+R\right)+M_{F} .
$$

If $\left(1-N_{1}\right) R \geq\left|y_{0}-t_{0}\right|$, then Eq. (14) has a solution in the function set

$$
\Phi_{N_{1}}=\left\{y \in C^{0}\left(\left[t_{0}-l_{1}, t_{0}+l_{1}\right]\right): y\left(t_{0}\right)=y_{0},|y(t)-y(s)| \leq N_{1}|t-s|, \forall t, s \in\left[t_{0}-l_{1}, t_{0}+l_{1}\right]\right\}
$$

by Theorem 4.1, where arbitrary $l_{1} \in\left[\left|y_{0}-t_{0}\right| /\left(1-N_{1}\right), R\right]$. In fact, for any $y \in \varphi_{N_{1}}$, we see that the function

$$
f\left(t, y^{[1]}(t), y^{[2]}(t), \ldots, y^{[m]}(t)\right)=\sum_{i=1}^{m} a_{i}(t) y^{[i]}(t)+F(t)
$$

is continuous on $\left[t_{0}-l_{1}, t_{0}+l_{1}\right]$ and

$$
\begin{aligned}
& \left|f\left(t, y^{[1]}(t), y^{[2]}(t), \ldots, y^{[m]}(t)\right)\right| \\
& \quad=\left|\sum_{i=1}^{m} a_{i}(t) y^{[i]}(t)+F(t)\right| \\
& \quad \leq \sum_{i=1}^{m} M_{a}\left(\left|t_{0}\right|+R\right)+M_{F} \\
& \quad=m M_{a}\left(\left|t_{0}\right|+R\right)+M_{F}=N_{1} .
\end{aligned}
$$

Since $\left(1-N_{1}\right) R \geq\left|y_{0}-t_{0}\right|$, the condition of Theorem 4.1 is satisfied, there exists a solution $y=\psi(t)$ of Eq. (14) in the functional set $\psi_{N_{1}}$. In the sequel, we show that $\psi^{(n+1)}(t)$ also is Lipschitzian on the compact interval $\left[t_{0}-l_{1}, t_{0}+l_{1}\right]$. In view of Lemma 5.1, we obtain

$$
\begin{aligned}
y_{* i k}(t) & =P_{i k}\left(y_{10}(t), \ldots, y_{1, i-1}(t) ; \ldots ; y_{k 0}(t), \ldots, y_{k, i-1}(t)\right) \\
& =P_{i k}\left(y^{\prime}(t), y^{\prime}\left(y_{1}\right), \ldots, y^{\prime}\left(y_{i-1}\right) ; \ldots ; y^{(k)}(t), y^{(k)}\left(y_{1}\right), \ldots, y^{(k)}\left(y_{i-1}\right)\right),
\end{aligned}
$$

where $y_{n}=y^{[n]}(t), n=1,2, \ldots, i-1$.

Denote

$$
\begin{aligned}
& H_{i k}=P_{i k}(\overbrace{N_{1}, \ldots, N_{1}}^{i \text { terms }} ; \overbrace{N_{2}, \ldots, N_{2}}^{i \text { terms }} ; \ldots ; \overbrace{N_{k}, \ldots, N_{k}}^{i \text { terms }}), \\
& a_{i}(t) \in \Omega\left(L_{i 1}, \ldots, L_{i(n+1)} ;\left[t_{0}-l_{1}, t_{0}+l_{1}\right]\right), \\
& F(t) \in \Omega\left(M_{1}, \ldots, M_{n+1} ;\left[t_{0}-l_{1}, t_{0}+l_{1}\right]\right) .
\end{aligned}
$$


Then, for any $t_{1}, t_{2} \in\left[t_{0}-l_{1}, t_{0}+l_{1}\right]$, we get

$$
\begin{aligned}
&\left|\psi^{(m+1)}\left(t_{1}\right)-\varphi^{(m+1)}\left(t_{2}\right)\right| \\
& \leq \sum_{i=1}^{n} \sum_{s=0}^{m} C_{m}^{s}\left|a_{i}^{(m-s)}\left(t_{1}\right)\left(\psi^{[i]}\left(t_{1}\right)\right)^{(s)}-a_{i}^{(m-s)}\left(t_{2}\right)\left(\psi^{[i]}\left(t_{2}\right)\right)^{(s)}\right| \\
&+\left|F^{(n)}\left(t_{1}\right)-F^{(n)}\left(t_{2}\right)\right| \\
& \leq \sum_{i=1}^{n}\left\{\left|a_{i}^{(m)}\left(t_{1}\right)-a_{i}^{(m)}\left(t_{2}\right)\right| \cdot\left|\psi^{[i]}\left(t_{1}\right)\right|+\left|a_{i}^{(m)}\left(t_{2}\right)\right| \cdot\left|\psi^{[i]}\left(t_{1}\right)-\psi^{[i]}\left(t_{2}\right)\right|\right\} \\
&+\sum_{i=1}^{n} \sum_{s=1}^{m} C_{m}^{s}\left(\left|a_{i}^{(m-s)}\left(t_{1}\right)-a_{i}^{(m-s)}\left(t_{2}\right)\right| \cdot\left|\left(\psi^{[i]}\left(t_{1}\right)\right)^{(s)}\right|\right. \\
&\left.+\left|a_{i}^{(m-s)}\left(t_{2}\right)\right| \cdot\left|\psi\left(\psi_{1,0}\left(t_{1}\right), \ldots, \psi_{s, i-1}\left(t_{1}\right)\right)-\psi\left(\psi_{1,0}\left(t_{2}\right), \ldots, \psi_{s, i-1}\left(t_{2}\right)\right)\right|\right) \\
&+M_{n+1} \frac{\left|t_{1}-t_{2}\right|^{\gamma}}{\Gamma(\gamma+1)} \\
& \leq \sum_{i=1}^{n}\left(L_{i(m+1)}\left(\left|t_{0}\right|+l_{1}\right)+L_{i m} N_{1}^{i}\right) \frac{\left|t_{1}-t_{2}\right|^{\gamma}}{\Gamma(\gamma+1)} \\
&+\sum_{i=1}^{n} \sum_{s=1}^{m} C_{m+1}^{s}\left(L_{i(m+1-s)} H_{i s}\left|t_{1}-t_{2}\right|+t_{i} \mid .\right. \\
&\left.+\sum_{u=1}^{s} \sum_{v=0}^{i-1} N_{u v}^{i s}\left|\psi_{u v}\left(t_{1}\right)-\psi_{u v}\left(t_{2}\right)\right|\right)
\end{aligned}
$$

Since

$$
\left|\psi_{u v}\left(t_{1}\right)-\psi_{u v}\left(t_{2}\right)\right| \leq N_{u+1}\left|\psi^{[v]}\left(t_{1}\right)-\psi^{[v]}\left(t_{2}\right)\right| \leq N_{u+1} N_{1}^{v} \frac{\left|t_{1}-t_{2}\right|^{\gamma}}{\Gamma(\gamma+1)},
$$

we attain

$$
\begin{aligned}
& \left|\psi^{(m+1)}\left(t_{1}\right)-\psi^{(m+1)}\left(t_{2}\right)\right| \\
& \leq \sum_{i=1}^{n}\left(L_{i(m+1)}\left(\left|t_{0}\right|+l_{1}\right)+L_{i m} N_{1}^{i}\right) \frac{\left|t_{1}-t_{2}\right|^{\gamma}}{\Gamma(\gamma+1)}+\sum_{i=1}^{n} \sum_{s=1}^{m} C_{m}^{s}\left(L_{i(m+1-s)} H_{i s}\left|t_{1}-t_{2}\right|\right. \\
& \left.\quad+L_{i(m-s)} \sum_{u=1}^{s} \sum_{v=0}^{i-1} N_{u v}^{i s}\left|\psi_{u v}\left(t_{1}\right)-\psi_{u v}\left(t_{2}\right)\right|\right)+M_{n+1} \frac{\left|t_{1}-t_{2}\right|^{\gamma}}{\Gamma(\gamma+1)} \\
& =\left\{\left(\sum_{i=1}^{n} L_{i(m+1)}\left(\left|t_{0}\right|+l_{1}\right)+L_{i m} N_{1}^{i}\right)\right. \\
& \left.\quad+\left(\sum_{i=1}^{n} \sum_{s=1}^{m} C_{m}^{s}\left(L_{i(m+1-s)} H_{i s}+L_{i(m-s)} \sum_{u=1}^{s} \sum_{v=0}^{i-1} N_{u v}^{i s} N_{u+1} N_{1}^{v}\right)\right)+M_{n+1}\right\} \frac{\left|t_{1}-t_{2}\right|^{\gamma}}{\Gamma(\gamma+1)},
\end{aligned}
$$

which implies that $\psi^{(m+1)}(t)$ is Lipschitzian.

Example 5.1 Consider the equation

$$
D^{\frac{1}{2}} y(t)=\frac{1}{5} y(y(t))-\frac{1}{4}
$$


associated with

$$
y(-1)=-\frac{1}{2}
$$

Now we check the conditions of Theorem 4.2. For the compact interval $[-1,0]$, we see that $y_{0}=-\frac{1}{2}, t_{0}=-1, \gamma=\frac{1}{2}, \Gamma\left(\frac{3}{2}\right)=0.88$ for all $t \in[-1,0]$. Since $M_{2}=\|y\|_{[-1,0]^{n+1}}=1 / 5+$ $1 / 4=9 / 20<0.5$, also $A_{t_{0}}=\max \left\{t_{0}-a, b-t_{0}\right\}=\max \{-1+1,0+1\}=\max \{0,1\}=1, B_{y_{0}}=$ $\min \left\{y_{0}-a, b-y_{0}\right\}=\min \left\{\frac{-1}{2}+1,0+\frac{1}{2}\right\}=\min \left\{\frac{1}{2}, \frac{1}{2}\right\}=1 / 2$, and $\Gamma\left(\frac{3}{2}\right)=0.88$, then $\frac{M_{2}}{\Gamma\left(\frac{3}{2}\right)}=\frac{9}{17.6}$, which satisfies the conditions of Theorem 4.2. Then Eq. (15) associated with Eq. (16) has a solution.

Remark 5.1 In the proof of an invariant set, they require the inequalities

$$
\begin{aligned}
& |(F x)(t)| \leq\left|x_{0}\right|+\left|\int_{0}^{t} f(s, x(s), x(x(s))) \frac{(t-s)^{\gamma}}{\Gamma(\gamma+1)} d s\right| \leq\left|x_{0}\right|+M \cdot \frac{\left|t-x_{0}\right|^{\gamma}}{\Gamma(\gamma+1)} \leq b, \\
& |(F x)(t)| \geq\left|x_{0}\right|-\left|\int_{0}^{t} f(s, x(s), x(x(s))) \frac{(t-s)^{\gamma}}{\Gamma(\gamma+1)} d s\right| \geq x_{0}-C_{x_{0}} \geq a .
\end{aligned}
$$

The right-most inequality of Eq. (15) contradicts the definition of $C_{x_{0}}$. We overcome this difficulty by defining $B_{y_{0}}$.

\section{Burton stability}

In this section, we aim to discuss the stability of solutions of a class of iterative fractional differential equations. We need the following observations.

Definition 6.1 Let $g:[0, \infty[\mapsto[1, \infty[$ be an arbitrary continuous strictly increasing function with $g(t) \mapsto \infty$ as $t \mapsto \infty$ and define a Banach space $\left(W,|\cdot|_{g}\right)$ of continuous functions $\Phi:\left[0, \infty\left[\mapsto \Re^{n}\right.\right.$ with the property that

$$
|\Phi|_{g}=\sup _{0 \leq t \leq \infty} \frac{|\Phi(t)|}{g(t)}<\infty .
$$

Definition 6.2 [24] Let $B$ be a closed and densely defined operator on $Y$ and $l \in \mathcal{C}\left(\Re^{+}\right)$ be a scalar kernel. A family $\mathfrak{\Im}_{t \geq 0}$ is called a $k$-regularized resolvent family if the following conditions are satisfied:

(a) $\mathfrak{\Im}$ is strongly continuous on $\mathfrak{R}^{+}$and $\mathfrak{\Im}(0)=k(0) I$;

(b) $\mathfrak{s} y \in D(B)$ and $B \mathfrak{s}(t)(y)=\mathfrak{s}(t) B y$ for all $y \in D(B)$ and $t \geq 0$;

(c) the $k$-regularized resolvent equation holds

$$
\mathfrak{\Im}(t)(y)=K(t)(y)-\int_{0}^{t} B K(t-s) \mathfrak{s}(s) y d s .
$$

Lemma 6.1 [25] Let $N$ be a closed ball in $A B$, let $P: N \rightarrow N$ be continuous, and let $P N$ be locally equicontinuous. Then there exists a point $\Psi \in N$ with $P \Psi=\Psi$.

Lemma 6.2 [25] Let $N$ be a convex, nonempty, bounded subset of $A B$, let $P: N \mapsto N$, and let $P N$ be locally equicontinuous. If, in addition, the g-norm closure of PN is in $N$, then there exists a point $\Psi \in M$ with $P \Psi=\Psi$. 
Next, we discuss the stability of Eq. (14) for a special case by putting

$$
F(t)=\sum_{i=1}^{m} B_{i}(t)(t) y^{[i]}(t-r(t))
$$

Theorem 6.1 Consider the fractional differential equation

$$
D^{\gamma} y(t)=\sum_{i=1}^{m}\left[-A_{i}(t) y^{[i]}(t)+B_{i}(t) y^{[i]}\left(t-r_{i}(t)\right)\right], \quad y(0)=y_{0}, \quad 0<\gamma<1,
$$

where $A_{i}, B_{i}, r_{i}:[0, \infty) \mapsto[0, \infty)$ are continuous. Moreover, suppose that

(a) $a_{i}:=-A_{i}(t), A_{i}(t)$ is bounded on $[0, \infty[$;

(b) $A_{i}(t)-B_{i}(t) \geq \delta$ for all $t_{0} \geq 0$, and a constant $\delta>0$;

(c) $r_{i}(t) \geq 0$ for all $t \geq 0$.

Then the zero solution of Eq. (18) is stable.

Proof Consider problem (18). Assume that $\exists \zeta>0$ and $\max _{i}\left[\sup _{t \geq 0} A_{i}(t)\right]<\zeta$ for all $t \geq 0$ such that

$$
K(t)=\frac{\zeta}{\Gamma(\gamma)} t^{\gamma-1}
$$

Then for the Lizama resolvent $\left(\Im_{\gamma}\right)$, which satisfies

$$
\Im(t)=K(t)-\int_{0}^{t} K(t-s) \Im(s) d s
$$

we have

$$
0 \leq \Im(t) \leq K(t), \quad t \Im(t) \rightarrow 0 \quad \text { as } t \rightarrow \infty \quad \text { and } \quad \int_{0}^{\infty} \Im(s) d s=1 .
$$

Rewrite Eq. (18) as follows:

$$
\begin{aligned}
D^{\gamma} y(t) & =\sum_{i=1}^{m}\left[-A_{i}(t) y^{[i]}(t)+B_{i}(t) y^{[i]}\left(t-r_{i}(t)\right)\right] \\
& =\sum_{i=1}^{m}\left[-\zeta y(t)+\zeta\left[y-y^{i}\right]+\left[\zeta-A_{i}\right](t) y^{[i]}+B_{i}(t)(t) y^{[i]}\left(t-r_{i}(t)\right)\right] .
\end{aligned}
$$

Therefore, the solution $y(t)$ of Eq. (18) achieves

$$
\begin{aligned}
y(t)= & \sum_{i=1}^{m}\left[w(t)+\int_{0}^{t} \Im_{\gamma}(t-s)\left[y-y^{[i]}\right](s) d s\right. \\
& \left.+\int_{0}^{t} \Im_{\gamma}(t-s)\left(1-\frac{A_{i}(t)}{\zeta}\right) y^{[i]}(s) d s+\int_{0}^{t} \Im_{\gamma}(t-s) \frac{B_{i}(t)}{\zeta} y^{[i]}\left(s-r_{i}(s)\right)\right]=:(P y)(t),
\end{aligned}
$$

where $w(t)=y_{0}\left(1-\int_{0}^{\infty} \Im(s) d s\right)$. 
Let $0<\epsilon<\frac{\sqrt{3}}{3}$, we may assume that $\zeta \geq 1$ and $0<\delta<1$ so that $\frac{\delta \epsilon^{3}}{\zeta}<\epsilon$. Now set

$$
n_{0}=\min _{i}\left[\inf \left\{s-r_{i}(s) ; s \geq 0\right\}\right]
$$

Let $\phi:\left[n_{0}, 0\right] \rightarrow \Re$ be a given continuous initial function with $\|\phi\|<\frac{\delta \epsilon^{3}}{\zeta}<\epsilon$. Define

$$
N=\{\Psi \in A B \mid\|\Psi\| \leq \epsilon\}
$$

For the natural mapping defined above with $y(s)=\phi(s)$ for $s \leq 0$, we can show that $P: N \mapsto N$. We observe that $\sum_{i=1}^{m}\left[r-r_{i}\right]$ is increasing on $[0, \epsilon]$ and we obtain

$$
\begin{aligned}
|(P y)(t)| & \leq \sum_{i=1}^{m}\left[|w(t)|+\left(\epsilon-\epsilon^{[i]}\right)+\epsilon^{[i]} \int_{0}^{t} \Im_{\gamma}(t-s)\left(1-\frac{A_{i}(t)}{\zeta}+\frac{\left|B_{i}(t)\right|}{\zeta}\right) d s\right] \\
& \leq \sum_{i=1}^{m}\left[|\phi(0)|+\left(\epsilon-\epsilon^{[i]}\right)+\epsilon^{[i]}\left(1-\frac{\delta}{\zeta}\right)\right]<\epsilon .
\end{aligned}
$$

It is clear that $P N$ is equicontinuous on $[0, \infty)$ and the $g$-norm closure of $P N$ is in $N$ (Lemma 6.1). Consequently, in view of Lemma 6.2, $P$ has a fixed point $y \in N$ which is a solution of Eq. (18). Thus, the zero solution of Eq. (18) is stable. This completes the proof.

\section{Competing interests}

The authors declare that they have no competing interests.

\section{Authors' contributions}

All authors jointly worked on deriving the results and approved the final manuscript.

\section{Author details}

${ }^{1}$ Institute of Mathematical Sciences, University of Malaya, Kuala Lumpur, 50603, Malaysia. ${ }^{2}$ Department of Mathematics, University Putra Malaysia, Serdang, Malaysia.

\section{Acknowledgements}

The authors would like to thank the referees for giving strong and very useful suggestions for improving the work.

Received: 28 November 2014 Accepted: 18 February 2015 Published online: 05 March 2015

\section{References}

1. Srivastava, HM, Owa, S: Univalent Functions, Fractional Calculus, and Their Applications. Halsted, New York (1989)

2. Miller, KS, Ross, B: An Introduction to the Fractional Calculus and Differential Equations. Wiley, New York (1993)

3. Kiryakova, V: Generalized Fractional Calculus and Applications. Longman, Harlow (1994); copublished in the United States with John Wiley \& Sons, Inc., New York

4. Podlubny, I: Fractional Differential Equations. Academic Press, San Diego (1999)

5. Hilfer, R: Application of Fractional Calculus in Physics. World Scientific, Singapore (2000)

6. West, BJ, Bologna, M, Grigolini, P: Physics of Fractal Operators. Springer, New York (2003)

7. Kilbas, AA, Srivastava, HM, Trujillo, Jj: Theory and Applications of Fractional Differential Equations. Elsevier, Amsterdam (2006)

8. Sabatier, J, Agrawal, OP, Machado, JA: Advances in Fractional Calculus: Theoretical Developments and Applications in Physics and Engineering. Springer, Berlin (2007)

9. Lakshmikantham, V, Leela, S, Vasundhara, DJ: Theory of Fractional Dynamic Systems. Cambridge Scientific Publishers, Cambridge (2009)

10. Klafter, J, Lim, SC, Metzler, R (eds.): Fractional Dynamics: Recent Advances. World Scientific, Singapore (2011)

11. Agarwal, RP, Ntouyas, SK, Ahmad, B, Alhothuali, MS: Existence of solutions for integro-differential equations of fractional order with nonlocal three-point fractional boundary conditions. Adv. Differ. Equ. 2013, 128 (2013)

12. Agarwal, RP, Arshad, S, O'Regan, S, Lupulescu, V: A Schauder fixed point theorem in semilinear spaces and applications. Fixed Point Theory Appl. 2013, 306 (2013)

13. Li, X, Han, Z, Sun, S, Zhao, P: Existence of solutions for fractional q-difference equation with mixed nonlinear boundary conditions. Adv. Differ. Equ. 2014, 326 (2014) 
14. Ibrahim, RW: Extremal solutions for certain type of fractional differential equations with maxima. Adv. Differ. Equ. 2012, 7 (2012)

15. Ibrahim, RW: Modified fractional Cauchy problem in a complex domain. Adv. Differ. Equ. 2013, 149 (2013)

16. Ibrahim, RW: On holomorphic solution for space- and time-fractional telegraph equations in complex domain. J. Funct. Spaces Appl. 2012, Article ID 703681 (2012)

17. Eder, E: The functional differential equation $x^{\prime}(t)=x(x(t))$. J. Differ. Equ. 54(3), 390-400 (1984)

18. Fečkan, M: On a certain type of functional differential equations. Math. Slovaca 43(1), 39-43 (1993)

19. Zhang, P, Gong, X: Existence of solutions for iterative differential equations. Electron. J. Differ. Equ. 2014, 7 (2014)

20. Ibrahim, RW: Existence of deviating fractional differential equation. CUBO 14, 127-140 (2012)

21. Ibrahim, RW: Existence of iterative Cauchy fractional differential equation. J. Math. 2013, Article ID 838230 (2013)

22. Ibrahim, RW, Darus, M: Infective disease processes based on fractional differential equation. In: Proceedings of the 3rd International Conference on Mathematical Sciences, vol. 1602, pp. 696-703 (2014)

23. Si, J-G, Wang, X-P: Smooth solutions of a nonhomogeneous iterative functional differential equation with variable coefficients. J. Math. Anal. Appl. 226(2), 377-392 (1998)

24. Lizama, C, Vergara, V: Uniform stability of resolvent families. Proc. Am. Math. Soc. 132(1), 175-181 (2004)

25. Burton, TA, Zhang, B: A Schauder-type fixed point theorem. J. Math. Anal. Appl. 417(2), 552-558 (2014)

\section{Submit your manuscript to a SpringerOpen ${ }^{\circ}$ journal and benefit from:}

- Convenient online submission

Rigorous peer review

- Immediate publication on acceptance

- Open access: articles freely available online

- High visibility within the field

- Retaining the copyright to your article 